\title{
RIPK1 prevents aberrant ZBP1-initiated necroptosis
}

\section{Tom Vanden Berghe and William J. Kaiser}

Receptor interacting protein kinase 1 (RIPK1) regulates inflammation and cell death, in host defense and homeostasis. The adaptor function of RIPK1 allows pro-survival and inflammatory signaling, while its kinase activity regulates the induction of necroptosis and apoptosis. A new level of regulation through its RIP homotypic interaction motif (RHIM) was recently discovered to suppress necroptosis and inflammation driven by the putative nucleic acid sensor protein Z-DNA binding protein 1 (ZBP1), also known as DAI.

In the two decades following the discovery of RIPK1, RIPK1 has emerged as a master regulator of inflammatory signaling and cell death [1]. To date, important advances in our understanding of RIPK1 function continue to be uncovered. RIPK1-deficient mice fail to thrive, die within 1-3 days following birth and display extensive apoptosis. These observations prompted initial research efforts to focus on the role of RIPK1 in controlling pro-survival and inflammatory gene expression. More recently, RIPK1 kinase activity emerged as essential for coordinating death receptor- induced necrosis (Figure 1A). This type of necrosis was dubbed necroptosis upon the discovery of a chemical kinase inhibitor of RIPK1, necrostatin-1 (Nec1). However, the model of RIPK1 kinase functioning exclusively for necroptosis proved too simplistic as kinase activity was also found to be crucial for caspase 8-mediated apoptosis under conditions where cellular inhibitors of apoptosis (cIAPs) are depleted (Figure 1A). Thus, depending on the cellular content, RIPK1 kinase can initiate either necroptosis or apoptosis. This implies that a protective phenotype observed in response to $\mathrm{Nec} 1$ or in the context of RIPK1 kinase dead knockin mice (Ripk1 ${ }^{\mathrm{KD}}$ mice) stems from the role of RIPK1 in regulating cell death but does not differentiate the cell death modality. Conditional deletion of RIPK1 in mice revealed additional complexity on the regulatory action of RIPK 1 . While absence of RIPK1 unleashes apoptosis in the intestinal epithelial cells $[2,3]$, necroptotic cell death dominates in the skin upon RIPK1 depletion [3]. RIPK1 kinase dead knock-in mice did not show any spontaneous phenotype indicating the sensitization to apoptosis in the gut upon RIPK1 deletion
A

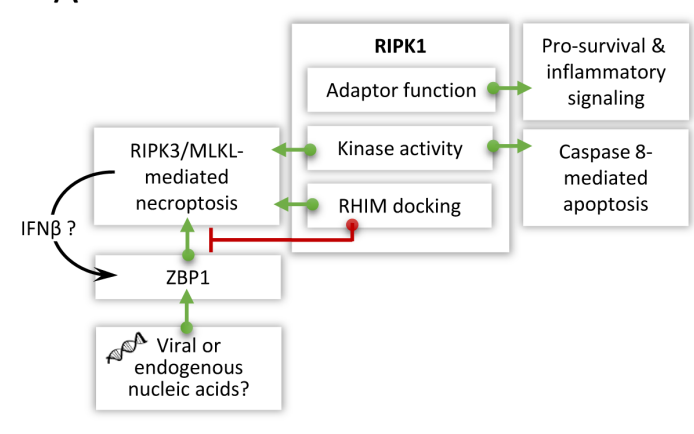

B

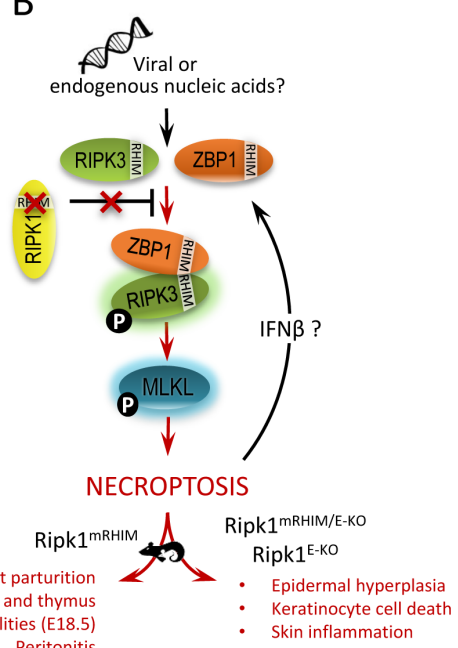

Figure 1: RIPK1 a master regulator of cell death and inflammation. A. Schematic representation of the versatile functions of RIPK1. Basically, RIPK1 regulates pro-survival and inflammatory signaling in addition to apoptotic and necroptotic cell death through its different regulatory domains viz. adaptor, kinase and RHIM docking function. B. Schematic representation of how the RHIM domain of RIPK1 acts as an essential break on constitutive ZBP1 activation. RIPK1 with an inactivating mutation in the RHIM that abolishes the capacity of RIPK1 to interact with other cellular RHIM containing proteins induces ZBP1/RIPK3/MLKL- mediated necroptosis and inflammation. This defect signaling results in a spontaneous phenotype in the mentioned RIPK1 transgenic mice, which are briefly described. IFN, interferon; RIPK, receptor interacting protein kinase; Ripk $1^{\text {mRHIM }}$, mice expressing RIPK1 with an inactivating mutation in the RHIM; Ripk1 $1^{\text {mRHIM/E-KO }}$, mice with skin specific expression of RIPK1 with an inactivating mutation in the RHIM; Ripk $1^{\mathrm{E}-\mathrm{KO}}$, mice with skin specific deletion of RIPK1; RHIM, RIP homotypic interaction motif; ZBP1, Z-DNA binding protein 1. 
or necroptosis in the skin was not due to the absence of kinase activity $[4,5]$. In the intestine, the absence the death domain in RIPK1 led to excessive apoptosis pointing to the essential scaffold function of this domain in RIPK1 for induction of pro-survival and inflammatory signaling (Figure 1A). However, the role of RIPK1 in preventing necroptosis in the skin remained unresolved until now.

In the current issue of Nature, both the Pasparakis and Dixit Labs independently reveal that the absence of RIPK1 in the skin promotes a necrotic phenotype driven by ZBP1, a RHIM-containing protein that has previously been shown to mediate virus-induced necroptosis through activation of RIPK3/MLKL [6, 7]. As a proof of concept, both groups generated mice expressing RIPK1 with an inactivating mutation (referred to as Ripk $1^{\mathrm{mRHIM}}$ mice) in the RHIM that abolishes the capacity of RIPK1 to interact with other cellular RHIM containing proteins. Strikingly, Ripk $1^{\text {mRHIM }}$ mice died around birth with enhanced cell death in the skin that correlated with robust levels of RIPK3 autophosphorylation. Blocking necroptosis with catalytically inactive RIPK3 D161N, RHIM mutant RIPK3, RIPK3 deficiency, or MLKL deficiency prevented lethality in Ripk $1^{\text {mRHIM }}$ mice. Importantly, the loss of ZBP1 prevented perinatal lethality in Ripk $1{ }^{\mathrm{mRHIM}}$ mice, unveiling an unexpected role for RIPK1 in preventing ZBP1 dysregulation. In line with these findings, ZBP1 deficiency also prevented the development of skin lesions in mice with skin-specific depletion of RIPK1 (Ripk1 $1^{\mathrm{E}-\mathrm{KO}}$ mice) or skin-specific expression of RIPK $1^{\text {mRHIM }}$ (Ripk $1^{\text {mRHIM/E-KO }}$ mice) [7]. Mechanistically, ZBP1 interacted strongly with phosphorylated RIPK3 in cells expressing RIPK1 ${ }^{\text {mRHIM }}$, suggesting that the RIPK1 RHIM may prevent ZBP1 from binding and activating RIPK3 (Figure 2B). However, both studies were unable to detect constitutive interaction between RIPK1 and ZBP1. Similar to RIPK3 knock out mice [1], elimination of ZBP1, but not the other cellular RHIM-containing protein TRIF, also rescued the perinatal lethality of RIPK1/Caspase 8-deficient mice [6]. In summary, at least three independent transgenic approaches underscore the suppressive role of RIPK1 on ZBP1induced necroptosis and inflammation.

What could be the reason of the death at birth of Ripk $1^{\text {mRHIM }}$ mice? Given that Ripk $1^{\text {mRHIM/E-KO }}$ mice are viable, the lethality of Ripk $1{ }^{\mathrm{mRHIM}}$ mice is unlikely driven by increased cell death and/or inflammation in the skin. It is tempting to speculate that in Ripk $1^{\mathrm{mRHIM}}$ mice, exposure to microbes after birth initiates lethal ZBP1/ RIPK3/MLKL-driven inflammation similar to systemic inflammatory response syndrome (SIRS). It is remarkable that, except for the skin, no significant increase in cell death was observed in Ripk $1^{\text {mRHIM }}$ mice. Undetectable levels of necroptotic cell death in cells other than the epidermis could be sufficient to initiate inflammation and consequent SIRS-induced shock. Additional research will need to distinguish whether ZBP1 functions as merely an interferon inducible RHIM-adapter protein or as a bona fide nucleic acid sensor surveilling RNA or DNA derived from microbes or even endogenous nucleic acids, perhaps from damaged tissue. Furthermore, additional studies will be necessary to resolve whether RIPK1 functions as an essential brake on constitutive ZBP1 activation or as a potential sink for RIPK3 limiting activation by RIPK1. Nonetheless, these two studies have illuminated an unexpected role for ZBP1 in inflammation and the importance of RHIM interactions in both driving as well as limiting cell death.

William J. Kaiser: Departments of Microbiology, Immunology and Molecular Genetics, University of Texas Health Sciences Center, San Antonio, TX, USA

Correspondence to: William J. Kaiser, email kaiserw@uthscsa.edu

Tom Vanden Berghe: Department of Biomedical Molecular Biology, Inflammation Research Centre, VIB, Ghent University, Ghent, Belgium

Correspondence to: Tom Vanden Berghe, email Tom.VandenBerghe@irc.vib-ugent.be

Keywords: necroptosis, RIPK1, ZBP1, RIPK3, MLKL, Autophagy

Received: December 05, 2016

Published: December 12, 2016

\section{REFERENCES}

1. Silke J, et al. Nat Immunol. 2015; 16:689-697.

2. Takahashi N, et al. Nature. 2014; 513:95-99.

3. Dannappel M, et al. Nature. 2014; 513:90-94.

4. Berger SB, et al. J Immunol. 2014; 192:5476-5480.

5. Polykratis A, et al. J Immunol. 2014; 193:1539-1543.

6. Newton K, et al. Nature. 2016; 540:129-133.

7. Lin J, et al. Nature. 2016; 540:124-128. 